\title{
DESIGNING AN ENERGY EFFICIENT Clustering in HeterogeneOUS WIRELESS SENSOR NETWORK
}

\author{
Lakshmi M and Prashanth C R \\ Department of Telecommunication Engineering, \\ Dr. Ambedkar Institute of Technology, Bangalore, India
}

\begin{abstract}
Designing an energy-efficient scheme in a Heterogeneous Wireless Sensor Network (HWSN) is a critical issue that degrades the network performance. Recharging and providing security to the sensor devices is very difficult in an unattended environment once the energy is drained off. A Clustering scheme is an important and suitable approach to increase energy efficiency and transmitting secured data which in turn enhances the performance in the network. The proposed algorithm Energy Efficient Clustering (EEC) works for optimum energy utilization in sensor nodes. The algorithm is proposed by combining the rotation-based clustering and energy-saving mechanism for avoiding the node failure and prolonging the network lifetime. This shows MAC layer scheduling is based on optimum energy utilization depending on the residual energy. In the proposed work, a densely populated network is partitioned into clusters and all the cluster heads are formed at a time and selected on rotation based on considering the highest energy of the sensor nodes. Other cluster members are accommodated in a cluster based on Basic Cost Maximum flow $(B C M F)$ to allow the cluster head for transmitting the secured data. Carrier Sense Multiple Access (CSMA), a contention window based protocol is used at the MAC layer for collision detection and to provide channel access prioritization to HWSN of different traffic classes with reduction in End to End delay, energy consumption, and improved throughput and Packet delivery ratio(PDR) and allowing the cluster head for transmission without depleting the energy. Simulation parameters of the proposed system such as Throughput, Energy, and Packet Delivery Ratio are obtained and compared with the existing system.
\end{abstract}

\section{KEYWORDS}

HWSN, Clustering, EEC, CSMA, MAC, PDR.

\section{INTRODUCTION}

Wireless sensor network (WSN)[1] is the group of sensor nodes that are deployed randomly in a focused area over actively changing environments. These wireless sensor nodes are used for sensing, processing, and forwarding the data to the intermediate nodes or neighboring nodes and to the Base Station (BS) [2]. Since these nodes are small devices and are limited with memory, low processing, low computation, and small power unit that are battery-powered.

The sensor nodes are scattered in a sensor field which are transceivers having the capability of collecting, routing the data to the sink using multihop infrastructure-less architecture. The devices carry computations using their processing capabilities to transmit the processed and required data. WSNs are used mainly in civilian applications such as detecting, monitoring enemy movements, nuclear and health care applications, chemical, biological, automation, tracking, and others. 


\subsection{Sensor Node}

There are five components in a sensor node; a microcontroller unit, a transceiver unit, a memory unit, a power unit, and a sensor unit as shown in Figure 1. The microcontroller unit is responsible for handling different tasks such as data processing and controlling other components in the sensor node.

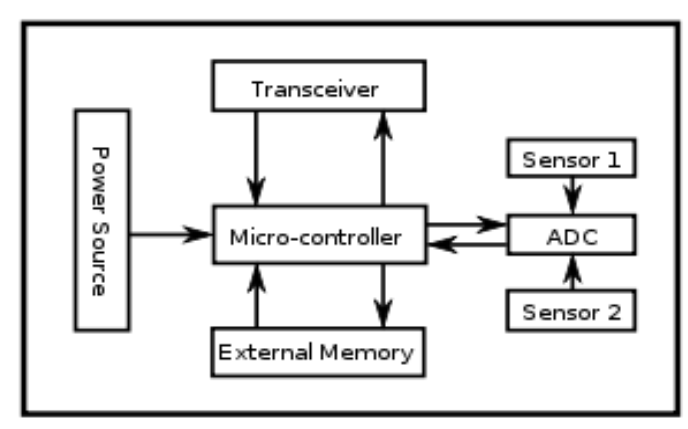

Figure 1: Components of a sensor node

The controller unit is having a small storage unit that is integrated into the embedded board or onboard memory. This unit also takes the risk of managing the procedures that make the sensor node for sensing, processing using algorithms, and collaborating with the other nodes using wireless communication. The transceiver unit is the most power-consuming unit where a sensor node communicates with other nodes and other parts of the WSN. Random Access Memory, Read-Only Memory and other memory types are used as temporary storage of sensed data. The power unit is a critical component for supplying node energy. Batteries are used for storing power. The sensor unit is the main component in a wireless sensor node which provides information-gathering capabilities from the physical phenomenon. This unit is responsible for gathering information from the outside world like light, sound, temperature, and others. There are two subunits i.e. a sensor and an analogue to digital converter (ADC).

It is known that Energy efficiency is the ratio of the total amount of data delivered to the total energy consumed. The energy efficiency [3] increases as more data is transmitted from the source to destination in a given amount of energy consumption. In turn, it is the one which provides the same service by using less energy. The focus of these techniques is to achieve energy efficiency and maximization of network lifetime. Clustering is the most widely used technique in hierarchical routing to achieve these parameters. Redundant messages are eliminated while forming efficient clusters and selecting or reselecting the Cluster Head $(\mathrm{CH})$. It is required to construct the topology for distributing the nodes uniformly in the clusters which makes the network efficient. Poor network performance is due to the excessive energy consumption while forming the clusters periodically and reselecting the $\mathrm{CH}[4,5$, and 6].

\subsection{Routing Protocols}

An algorithm for defining the exact route for a packet from source to destination is nothing but the routing protocol. Due to resource constraints in WSN, routing is more challenging compared to mobile ad-hoc networks or vehicular ad-hoc networks [7]. So new routing mechanisms are very important in keeping in view the network architecture and application requirements. High energy consumption results due to negligence in route maintenance and frequent topological changes. Researchers have found various routing techniques for minimizing energy consumption and maximizing the network lifetime. The categories of routing protocols are shown in Figure 2. 


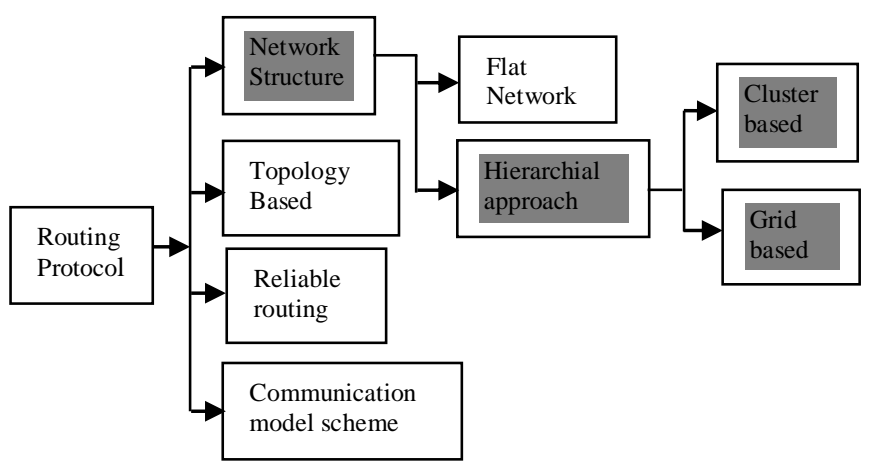

Figure 2: Classification of routing protocols

Nodes in the network are grouped into clusters by using some algorithms and the cluster head is elected on some criteria and held responsible for routing the packets. Two-layer approaches are used in hierarchical routing and one layer is for sensing the physical phenomenon and other layers for routing. Higher energy nodes are used for collecting, aggregating, and forwarding the data sensed by lower energy nodes [8]. Scalability and effective communication can be achieved through clustering approaches. These approaches have many advantages such as Data aggregation efficiency, efficient usage of channel bandwidth, and increase in scalability.

Some of the clustering techniques are compared such as Low Energy Adaptive Clustering Hierarchy(LEACH), Hybrid Energy Efficient Distributed Protocol(HEED), Extended HEED, Distributed Weight-based Energy-efficient Hierarchical Clustering protocol (DWEHC), Algorithm for Cluster Establishment (ACE).

\subsection{Energy Efficiency in Mac Protocols in WSN}

A significant amount of energy is saved in a multi-hop network by the occurrence of routing using an efficient routing algorithm. Transmission power is dependent based on distance and range, and energy is a major factor while route discovery. Sensor node has a component called Radio which is a major power-consuming and it is controlled by Medium Access Control (MAC) Protocol. Network lifetime increases to a great extent by an efficient MAC Protocol. Besides, the sharing of the wireless mediums is also controlled by the MAC protocol [9]. Less power is consumed by a sensor node when it is in sleep mode.

There are three types of traffic in IEEE 802.15.4 standard such as periodic, intermittent, and low latency data. Guaranteed access of the medium is required for allocating the time slots. One of the critical application securities monitoring which defines the repetitive low data. There are two modes in which the MAC protocol provides for three types of traffic such as beacon-enabled and non-beacon-enabled modes. Beacon enabled mode is for stat topology that defines the Guaranteed Time Slot(GTS) in a superframe. Peer to peer network is used for non beaconed enabled mode which is self-organized but does not acknowledge where the data is delivered or not. CSMA/CA is compared for the analysis of this contention free allocation of GTS. The MAC layer uses the medium in two different modes. The modes allow the frame transmission through a physical channel. A beacon-enabled mode uses CSMA/CA mechanism and offers GTS in a superframe used mostly for time-critical applications. MAC layer provides guaranteed services, controlling of frame validation, and security services.

One of the adaptive protocols called Sensor Protocols for Information via Negotiation (SPIN) [10] assumes all the nodes as potential base stations and disseminates all the information at each 
node to all other nodes. This protocol having variants are proposed to enhance the performance of the network. In Gradient Based Routing (GBR)[11], the difference between the height of a node and its neighbor node is calculated named as gradient value. Transmission of packet takes place on a link having the largest gradient value.GBR working is based on memorizing hops count while diffusing the entire network. Some authors have proposed a hierarchial routing algorithm which is energy-aware routing for cluster-based networks [12]. This method is based on the architecture of three tiers. Here the clusters are formed by grouping the sensors and Timedivision multiple access (TDMA) MAC scheme is used to operate the network.

Medium Access control protocols are very important in wireless communication for accessing the channel. Designing MAC protocols have many issues such as collision, overhearing, and terminal problems. Since sensors are battery-powered, energy is very important for sensor nodes. Energy consumption is more in the case of collision, data duplication, delay, and quality of service in MAC protocol design. Contention based protocol is a communication protocol used to operate telecommunication equipment in a wireless environment where many users use the same radio channel without coordination. MAC protocols are divided into TDMA protocols, CSMA based protocols, and Hybrid protocols. Sensor nodes wake up periodically in CSMA based protocols, listen to WSN, and again back to sleep. Using CSMA, throughput can be achieved at a lower traffic load.

The network flow approach [13] is introduced to overcome the problem of routing in sensor networks. The approach is better in maximizing the network lifetime by considering the link cost as a function remaining energy of the nodes and the transmission energy using the link.

\subsection{Motivation}

Some issues are found in the clustering protocols such as a selection of faithful $\mathrm{CH}$, Formation of energy-efficient clusters, and management of the network. The probabilistic approach is considered and linked to the criteria while selecting the $\mathrm{CH}$. A node can be selected as $\mathrm{CH}$ by varying the selection of $\mathrm{CH}$ which in turn increases the internal overhead. Energy consumption in the network is increased when the node is selected as $\mathrm{CH}$ in the region and hence the performance of the network reduces. All the nodes connected in the network should have better internal parameters and connectivity. So the performance parameters are enhanced by selecting the better $\mathrm{CH}$ approach and transmitting secured data.

The work is contributed to overcoming the issues of energy consumption and secured data transmission. MAC layer has a scheduling mechanism for designing a network that makes collision-free and more energy conserving. Many MAC protocols have been designed to avoid collision on a medium and contention window based protocol CSMA plays an important role during data transmission which occurs while carrier sensing. In the network, clusters are formed and $\mathrm{CHs}$ of all the clusters are selected at a time based on highest energy. The main idea is to select the $\mathrm{CH}$ for data transmission and other $\mathrm{CH}$ with less transmission and make them sleep. This methodology helps in achieving network lifetime by less consumption of node energy.

\section{LiTERATURE SURVEY}

A.Razaque et al., [14] have introduced an energy-efficient medium access control (EE-MAC) protocol for WSNs. Idle listening and congestion problem is handled for energy preservation. Semi synchronous low duty cycle is used in the protocol which uses less time for channel access. Authors have shown that higher throughput can be achieved using this. They have compared with existing protocols and achieved by saving $2 \%$ to $4.5 \%$ energy. 
Mohamed et al.,[15] have proposed a cluster-based routing protocol that has a two-tier structure. This has the benefit of combining cluster head distribution and chain based routing for minimizing the $\mathrm{CH}$ loss which occurs during data aggregation and transmission from Region of interest to base station. Adaptive length of time has been used reconstruct to the network for maintaining the adaptability with minimal network overhead. Authors have compared the work with other existing routing protocols and achieved a $100 \%$ increase in the network stability period. Accha et al.,[16] have studied the characteristics of MAC protocols for wireless sensor networks and observed one of the Sensor MAC's energy efficiency over CSMA/CA and simulated using ns-2 over an ad-hoc wireless network. The authors analyzed the performance of different MAC protocols and selected a specific MAC protocol for application need.

Gangbam et al.,[17] have studied that the energy consumption by the $\mathrm{CH}$ during transmission with a base station is higher compared to the energy depleted by cluster members to transmit the data to the respective $\mathrm{CHs}$. Authors proposed a protocol that organizes the selection of $\mathrm{CH}$ efficiently for providing the network lifetime and also balancing the $\mathrm{CH}$ selection. They have achieved better performance compared to other protocols. Liu et al., [18] proposed an algorithm called a novel Quorum Time Slot Adaptive Condensing (QTSAC) based medium access control for minimizing the delay and energy efficiency of a wireless sensor networks. A duty cycle of the system is prolonged by condensing the Quorum time slot to the data transmission period based on WSN characteristics and also this innovation reduced the network latency. Utilization of energy remaining in the area which is far from the sink increases the QTS for achieving the abovementioned parameters. S A Sert et al., [19] proposed a two-tier distributed fuzzy logic-based protocol (TTDFP) for the data aggregation efficiency improvement in multihop wireless sensor networks. Clustering is considered for efficient aggregation requirements like consumed energy. A network is divided into clusters and data is transmitted from member nodes to the $\mathrm{CHs}$, then packets are received at the base stations from CHs. This proposed TTDFP for extending the lifespan of multihop WSNs by considering the clustering efficiency and routing phases. Also, tuning of parameters is done by using the optimization framework used in fuzzy clustering.

Alsaafin et al., [20] designed a system by Heterogeneous Aware Distributed Clustering (HADC) algorithm. They presented node heterogeneity in terms of energy for enhancing the network lifetime. $\mathrm{CHs}$ in the proposed system are selected based on the cost function by considering the residual energy and node load. By making the trade-off between the distance and node degree towards $\mathrm{CHs}$, the authors reduced the energy consumption and balancing the load in the network. Proposed system performance is compared with HEED and LEACH in terms of efficiency. An S Toor et al.,[21] proposed a routing protocol named Energy Aware Cluster Based Multihop(EACBM) which reduces the energy consumption of sensor nodes by considering the concept of clustering and multihop communication. Additionally, the sub clustering concept is considered in the proposed system for the sensor nodes which are not reachable and are not included in any of the clusters. This protocol is compared with the existing protocols LEACH, CEEC, and SEP). Authors improved the network lifetime, stability and energy efficiency in heterogeneous WSNs.

R Lakshminarayanan et al.,[22] introduced a heterogeneous networking model based on two parameters that are primary and secondary. Primary is the one in which heterogeneity level is decided and secondary decides based on heterogeneity level. These are used in finding the residual energy of the cluster heads. In this model, the node in the heterogeneous network has a finite energy level based on the value of parameters. Evaluation of HEED protocol is done and Multi-level HEED is also tested. The proposed system reduces the energy dissipation and increases the network lifetime. C U Kumari et al., [23] have investigated a protocol for minimizing energy consumption in enhancing the stability period and network lifetime. For different scenarios in a heterogeneous network, Distributed Energy Efficient Clustering (DEEC) and Threshold Distributed Energy Efficient Clustering (TDEEC) are considered. Comparison of 
the above protocols is done by varying the advanced nodes, super nodes, and fraction of both to achieve network lifetime and stability period. Table I consists of some of the existing systems with achievements and drawbacks.

S A Almogahed et al.,[24] have proposed a scheme in heterogeneous WSNs for prolonging the network lifetime and stability period namely Optimized Distributed Energy Efficient Clustering (ODEEC). This protocol modifies the probability function of heterogeneity and the cluster head selection is optimized. In the proposed system, the network has two regions, the inner region, and outer region, also cluster head selection is not based on the position of BS distance nor the probabilities of heterogeneity. Authors achieved better performance in throughput, stability period and network lifetime. B El Bhiri et al., [25] have proposed the Efficient Routing Protocol for HWSN namely ERP-HWSN. In the proposed system, the cluster in the network has the highest inter correlated sensors through which the spectral graph theory is considered in an unbalanced graph. The result is to show the enhancement in network lifetime and compared with DEEC.

\subsection{Energy Consumption Model}

Transmitter and Receiver are the main sections of communication. Energy is required for both the sections, a sensor node having a transmitter section needs energy to run some circuits such as radio electronics and amplifier. Whereas energy consumed by the receiver section in the packet over a distance at radio electronics. The calculation of energy consumed is based on the free space energy consumption model as the distance between the communication entities is less than the threshold distance. If the distance is more than the threshold distance, then the multipath model is used. Data aggregation is a feature for the consumption of energy which is implemented to $\mathrm{CH}$ and the data collected by the cluster members is delivered to BS [26]. Energy consumed in the system is calculating by the parameters $L_{p}$, a packet of length (number of bits), and distance $d_{s}$ as shown in (1).

There are many significant parameters for processing the information. $\mathrm{E}_{\mathrm{el}}$ is the consumed energy by transmitting or receiving electronics circuitry. Two energies abbreviated as $E_{T}$ and $E_{R}$, are the transmitting and receiving energies consumed for processing a data packet $L_{P}$. A scheme of digital coding and modulation is used on which the parameters depend. Other parameters considered in the system are $\mathrm{E}_{\mathrm{fsm}}$ and $\mathrm{E}_{\mathrm{mpm}}$, the energy needed for the free space model, and multipath model.

For calculating energy, the free space power loss model is considered if the distance $d$ between the source and destination is less than $\mathrm{d}_{0}$ controlled by the power amplifier with the information. Transmitter with free space model need energy which is given as

$E_{T}\left(L_{p}, \mathrm{~d}_{\mathrm{s}}\right)=L_{p} E_{e l}+L_{p} E_{f s m} \mathrm{~d}_{\mathrm{s}}{ }^{2} \quad \mathrm{~d}_{\mathrm{s}} \ll<d_{0}$

Energy calculation using multipath model is given below in which distance $d_{s}$ is greater than the threshold distance.

$E_{T}\left(L_{p}, d_{s}\right)=L_{p} E_{e l}+L_{p} E_{m p m} d_{s}^{4} \quad d_{s}>>d_{0}$

Consumption of energy by the Receiver is,

$E_{R}\left(L_{p}, d_{s}\right)=L_{p} E_{e l}$

$d_{0}$ can be calculated as, 
$d_{0=} \sqrt{\mathrm{E}} \mathrm{fsm}_{\mathrm{fm}} / \sqrt{ } \mathrm{E}_{\mathrm{mpm}}$

\section{EEC NETWORK MODEL}

EEC is an energy-efficient clustering in the proposed network model which has sensor nodes deployed randomly on $\mathrm{NxN}$ sensing layout and uses heterogeneity with three-level node energy. After deployment, all the nodes and BS become static which is predefined. Links between each node for communication is considered to be static. $\mathrm{CH}$ is responsible in the sensing network for forwarding the data collected to BS directly. Data messages that are supervised or unsupervised transacted through wireless links. The assumptions made in the network include, the similar capabilities possessed by the nodes have unique id and different energy levels. The network is divided into clusters based on the node population and has BS is stationary and located at the middle of the network field. BS has a stable power supply without any memory restraints, energy, or computation.

In the network, every node is capable of sensing, aggregating, and forwarding the data. It is considered primarily that all the nodes have data to transfer always. Data aggregation is done by the nodes which compress the multiple data packets into a single packet. Energy transmission is regulated by the nodes with the receiving node distance and node failure is considered during the node deletion.

\subsection{Cluster Formation and Cluster Head Election}

Clustering is a grouping of nodes having similar capabilities. The process of clustering and information transmission consists of three stages such as Cluster arrangement and cluster head selection, accumulating information, collecting and transmitting the information. Based on residual energy of all the nodes, cluster head is selected and node density as per the protocol. Residual energy is defined as the total remaining energy and its node density which can be calculated within the sensing range. The highest residual energy node is chosen as a cluster head, which transmits the data across the cluster head. All the sensor nodes receive a broadcast messages by their respective cluster heads. Sensor nodes select the cluster head based on the received signal energy and based on the maximum received energy the decision is sent to $\mathrm{CH}$. Acknowledgment is sent to all sensor nodes regarding the CHs decision. Generation of TDMA signal and broadcasting is scheduled once the clusters are determined.

Finally, the energy levels of all the nodes are checked for proceeding to the next round and the clusters having zero energy are removed from the network. After forming the clusters, the cluster members in each cluster gather information and send it to their respective $\mathrm{CH}$. Once the data is collected by the $\mathrm{CH}$, the information transmission stage occurs by sending the aggregated data to the base station. The individuals in the group spend energies for gathering and sending the information to their $\mathrm{CHs}$.

\subsection{Formation of Cluster Head and Rotation}

Energy required for completing one round by $\mathrm{CH}$ is estimated as,

$E\left(\mathrm{CH}_{\text {load }}, \mathrm{R}_{-}\right)=C \mathrm{CH}_{(E l, R l, k)}+\mathrm{CH}(E l, A l, N C M)+C H_{(E l, T l, d l B S, k)}$

In the above equation, $\mathrm{E}_{1}, \mathrm{R}_{1}, \mathrm{~T}_{1}, \mathrm{~A}_{1}$ and $\mathrm{N}_{\mathrm{CM}}$ abbreviate energy, receive aggregation and cluster member nodes in a respective cluster in the first round of transmission. $\mathrm{E}\left(\mathrm{CH}_{\text {load }}, \mathrm{R}_{1}\right)$ is the 
energy required by $\mathrm{CH}$ for completing one round of transmission. $\mathrm{CH}_{(\mathrm{E} 1, \mathrm{R} 1, \mathrm{k})}$ is the energy consumed by $\mathrm{CH}$ for receiving the k-bits message. $\mathrm{CH}$ ( $\mathrm{E} 1, \mathrm{Al}, \mathrm{NCM})$ stands for energy needed to aggregate the data from Cluster members $\mathrm{N}_{\mathrm{CM}} . \mathrm{CH}{ }_{(\mathrm{E} 1, \mathrm{~T} 1 \mathrm{~d} 1 \mathrm{BS}, \mathrm{k})}$ indicates the consumption of energy to transmit the aggregated data to the base station.

$C H(E l, R l, N C M)=N_{C M} X\left(k X E_{e l}\right)$

$\mathrm{CH}(\mathrm{E} 1, \mathrm{~A} 1, \mathrm{NCM}+1)=\left(N_{C M}+1\right) X\left(k X E_{A}\right)$

$\left.C H_{(E l, T l, d l B S, k)=k X E_{e f_{+}} k X \in_{f s m X} d_{l}^{2} B S} \begin{array}{ll}\text { if } d_{1 B S}<d \\ k X \in_{m p m X} d_{l}^{4}{ }_{B S} & \text { otherwise }\end{array}\right\}$

$\mathrm{d}$ has the threshold value.

Pre-requisites:

1. All nodes are updated with neighbor information which is location aware.

2. Deployment of nodes is done randomly.

3. Measure the clusters of sensor nodes in the area, cost of communication such as initial energy levels and Probabilities of $\mathrm{CH}$.

4. Neighbor sensors are found within the sensing range of clusters.

5. All the neighboring nodes should be updated with some information such as initial energy level, residual energy of sensor nodes, $\mathrm{CH}$ probability and highest energy.

6. Cluster head selection is done by setting the probability.

7. In every round, nodes in each cluster are having data packets for transmission.

8. Attributes of all the nodes from each cluster will be sent to BS.

9. BS receives the information and updates and stores in the database.

10. Number of communication round completed successfully is estimated by $\mathrm{CH}$ before it dies.

The above equations (6), (7), and (8) calculate the energy required for receiving the data from the members in the cluster, aggregating the data with its data, and sending the aggregated data to the BS. $\mathrm{CH}$ does the estimation of the number of communication rounds before it dies.

The estimation of the number of communication round is done by $\mathrm{CH}$ successfully before it dies and given as,

$$
\mathrm{CH}_{\mathrm{E}_{-} \mathrm{R}}=\left\{\text { floor }\left(\mathrm{E}_{\mathrm{iE} \_\mathrm{CH}}\right) / E\left(\mathrm{CH}_{\text {load }}, R_{-}\right)\right\}
$$

In the above equation (9), $\mathrm{CH}_{\mathrm{E}_{-} \mathrm{R}}$ is the number of rounds that $\mathrm{CH}$ completes before it dies, and $\mathrm{E}_{\mathrm{iE} \_\mathrm{CH}}$ is the initial energy when the node becomes $\mathrm{CH}$ respectively.

Death of the $\mathrm{CH}$ is calculated as $=\mathrm{E}_{\mathrm{Ti}} / \mathrm{E}_{\mathrm{Ri}}$, where $\mathrm{E}_{\mathrm{Ti}}$ is the total energy and $\mathrm{E}_{\mathrm{Ri}}$ is the energy required to complete one round by $\mathrm{CH}$.

The dynamic threshold for $\mathrm{CH}-\mathrm{Rot}$ is calculated by using equation (9) by estimating $\mathrm{CH}_{\mathrm{E}_{\_} \mathrm{R} \text {. If }}$ $\mathrm{CH}_{\mathrm{E}_{\_} \mathrm{R}}>1$, then $\mathrm{CH}_{\mathrm{E}_{-} \mathrm{R}}-1$ is completed successfully by $\mathrm{CH}$ and again $\mathrm{CH}-\mathrm{Rot}$ is triggered for selecting $\mathrm{CH}$. The $\mathrm{CH}$ is selected among cluster members based on residual energy. If $\mathrm{CH}_{\mathrm{E}_{\_} \mathrm{R}}<1$, then re-clustering is done. Cluster head rotation is given below in equation (10),

$$
\mathrm{CH}-\mathrm{Rot}=\left\{\begin{array}{cc}
\mathrm{CH}_{\mathrm{E} \_\mathrm{R}}-1 & \text { if } \mathrm{CH}_{\mathrm{E} \_\mathrm{R}}>1 \\
\text { Re-clustering } & \text { Otherwise }
\end{array}\right\}
$$




\section{Proposed Protocol}

In this approach, energy-efficient clustering algorithm is introduced which consists of the following stages: Arrangement of clusters, Decision of group head, Accumulation of information and collection, and transmission of information It is necessary for selecting CHs based on initial energy, residual energy, and one hop count. All the nodes of different energy levels are deployed randomly over a network. Clusters are formed based on single-hop distance and neighbors are calculated using the Euclidian distance formula. Energy levels of each node are calculated as explained in the Energy consumption model. Once the energy levels of all the nodes are calculated, first $\mathrm{CH}$ is found using high initial energy. In the next step, all other $\mathrm{CH}$ are calculated based on the energy and also by giving a condition of not including the $\mathrm{CH}$ already found in other clusters. Totally $6 \mathrm{CHs}$ are found in the network. During transmission, a particular cluster is considered; $\mathrm{CH}$ is transmitting the data packets to the BS. Whereas other $\mathrm{CHs}$ remain static as the transmission is not taking place within those clusters. Once the energy of $\mathrm{CH}$ that is transmitting the data gets depleted, $\mathrm{CH}$ rotation takes place.

The scheme improves the utilization of energy and enhancing the remaining energy in the network. Contention window-based protocol CSMA/CA at the MAC layer enhanced for collision detection and avoidance for improving the faster transmission. Existing energy-efficient clustering protocols have issues such as Cluster size inconsistency, and location of selected $\mathrm{CH}$ towards the network boundary, and energy dissipation, affecting the network performance. After the cluster round, the remaining energy of a node must be high enough to handle the role of $\mathrm{CH}$ in the next upcoming rounds.

\section{Simulation Parameters}

The parameters set for implementation are highlighted below and simulation work having performance validation is explained.

Throughput: The amount of throughput per cluster round is defined as the number of data sent towards the base station from the sensor nodes over the cluster round. So it is the total number of data packets delivered at the destination node per unit time. It is measured in packets/second or bits/second.

Energy Efficiency: Energy consumed per unit of successful communication is referred to as Energy efficiency. It is defined as the ratio of throughput to the energy consumed.

Packet Delivery Ratio (PDR): PDR is the ratio of data packets delivered successfully to a destination to the total number of data packets sent by the sender. It can be calculated by considering the number of data packets sent and the number of received data packets.

End to End Delay:

The time taken for a data packet for transmission across a network from source to destination is referred to as End to end delay. It differs from Round trip time (RTT) and it is common in Internet Protocol network monitoring. The set of parameters utilized in implementing the proposed work for the simulation is given in Table 1 . 
Table 1: Simulation Parameters

\begin{tabular}{|l|l|l|l|}
\hline SI.No. & $\begin{array}{l}\text { Parameter } \\
\text { symbol }\end{array}$ & Description & Value \\
\hline 1 & $N \times N$ & Network area & $1300 \mathrm{~m}^{*} 1300 \mathrm{~m}$ \\
\hline 2 & $\mathrm{~N}$ & Number of nodes & 100 \\
\hline 3 & MAC & MAC protocol & IEEE 802.11 \\
\hline 4 & $E 0$ & Initial energy of nodes & $0.5-1.5 \mathrm{~J}$ \\
\hline 5 & $L_{p}$ & Data packet size & $4000 \mathrm{bits}$ \\
\hline 6 & $E_{\mathrm{el}}$ & $\begin{array}{l}\text { Radio electronics } \\
\text { energy }\end{array}$ & $50 \mathrm{~nJ} / \mathrm{bit}$ \\
\hline 7 & $E_{\mathrm{fsm}}$ & Free space energy & $10 \mathrm{pJ} / \mathrm{bit} / \mathrm{m} 2$ \\
\hline 8 & $E_{\mathrm{mpm}}$ & Amplification energy & $0.0013 \mathrm{pJ} / \mathrm{bit} / \mathrm{m} 4$ \\
\hline 9 & $d_{0}$ & Threshold distance & $87-87.7 \mathrm{~m}$ \\
\hline 10 & Traffic & Traffic Type & CBR \\
\hline 11 & Packet Size & Packet Size & $512 \mathrm{bytes}$ \\
\hline 12 & BS & Sink node & $(145,125)$ \\
\hline
\end{tabular}

\section{DISCUSSION}

In this section, the implementation and results of the simulated work and the validation with protocols like HEED and Rotating Energy Efficient Clustering for Heterogeneous Devices (REECHD) concerning parameters set shown in Table II are focus on. In a Heterogeneous Wireless Sensor Network, nodes are deployed randomly and the network is divided into clusters based on the population of nodes. BS is placed at the center of the network and node 49 with position $(145,125)$. A Number of nodes in the network is 100 , and all the nodes are assigned with different energy levels.

\subsection{Validation with HEED Protocol}

HEED [27] is a clustering protocol that produces equal-sized clusters. This algorithm has two phases, clustering and network operation. In the clustering phase, $\mathrm{CH}$ is selected based on residual energy, and member nodes that are close to the first $\mathrm{CH}$, join the cluster. Data packets get delivered from member nodes to BS in the operation phase. These two phrases get repeated over time. This protocol prevents two nodes from becoming $\mathrm{CHs}$ within the same transmission range. The Energy of sensor node gets depleted which is closest to the BS compared to the node that is farther away.

\subsection{Validation with REECHD Protocol}

REECHD [28] is a clustering protocol that uses equal size clustering and rotation. For leader election, this protocol combines node residual energy and node induced work. Intra traffic communication is reduced by the strategy of cluster head selection by prolonging the lifetime of the network. It defines the intra traffic communication limit accompanied by all clusters.

\subsection{Simulation Results}

\subsubsection{Network Initialization}

The proposed work uses the EEC algorithm having two phases, clustering phase, and operation phase. The network is densely populated with 100 nodes having different energy levels. As 
shown in Figure 3, all the nodes are placed randomly in a network area. In a flat grid. The parameters such as antenna type, link layer, queue type, routing protocol, and interface type are set up.

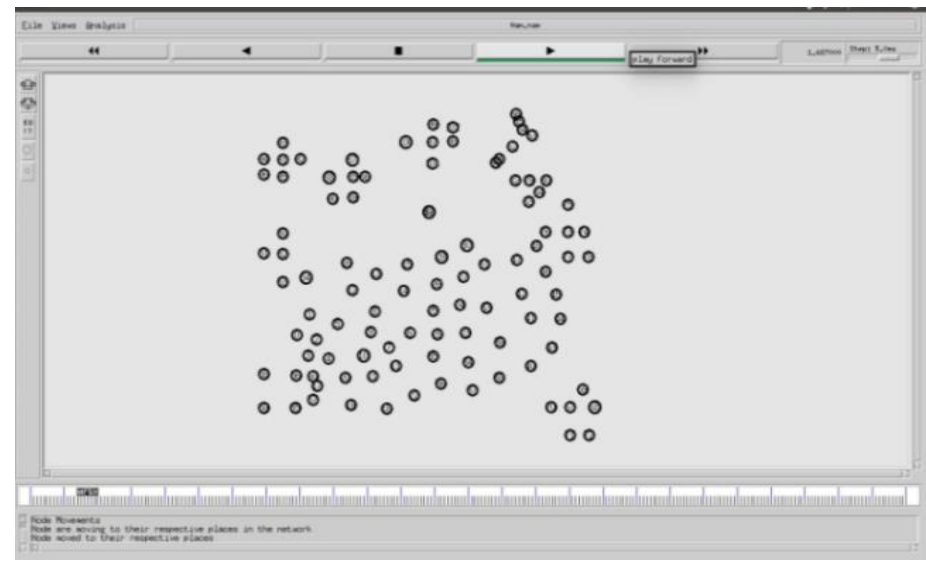

Figure 3: Node deployment

\subsubsection{Neighbor discovery}

As shown in Figure 4, the neighbor nodes are found out by Euclidian distance by considering the position of nodes i.e. (x, y) coordinates. The transmission range is set to $200 \mathrm{~m}$ and the nodes that fall in those ranges are considered. Each node should send Hello messages to find the position of nodes.

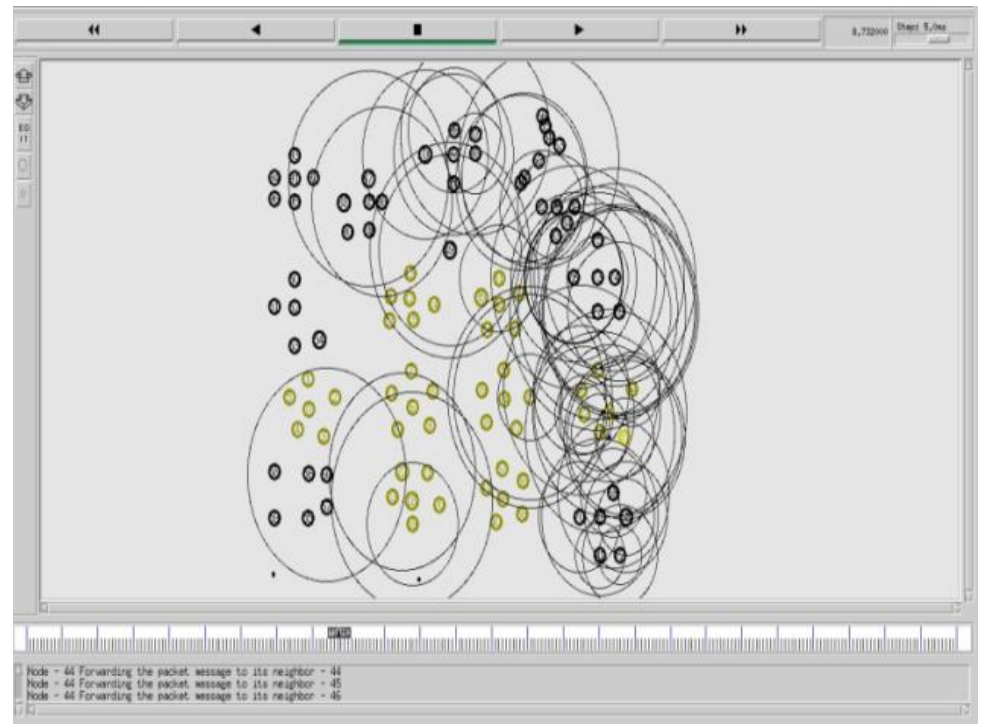

Figure 4: Neighbor Node Discovery

\subsubsection{Cluster Formation and $\mathrm{CH}$ selection}

The Clusters are formed based on one-hop distance as shown in Figure. 5 which are equally sized clusters. 100 nodes are deployed and 16 clusters are formed. Based on the high energy of nodes, $\mathrm{CH}$ is selected and other member nodes in the cluster have the feature of BCMF. 
International Journal of Computer Networks \& Communications (IJCNC) Vol.13, No.1, January 2021

Communication overhead of $\mathrm{CH}$ is reduced by these $\mathrm{CMs}$ as the maximum flow of data packets takes place.

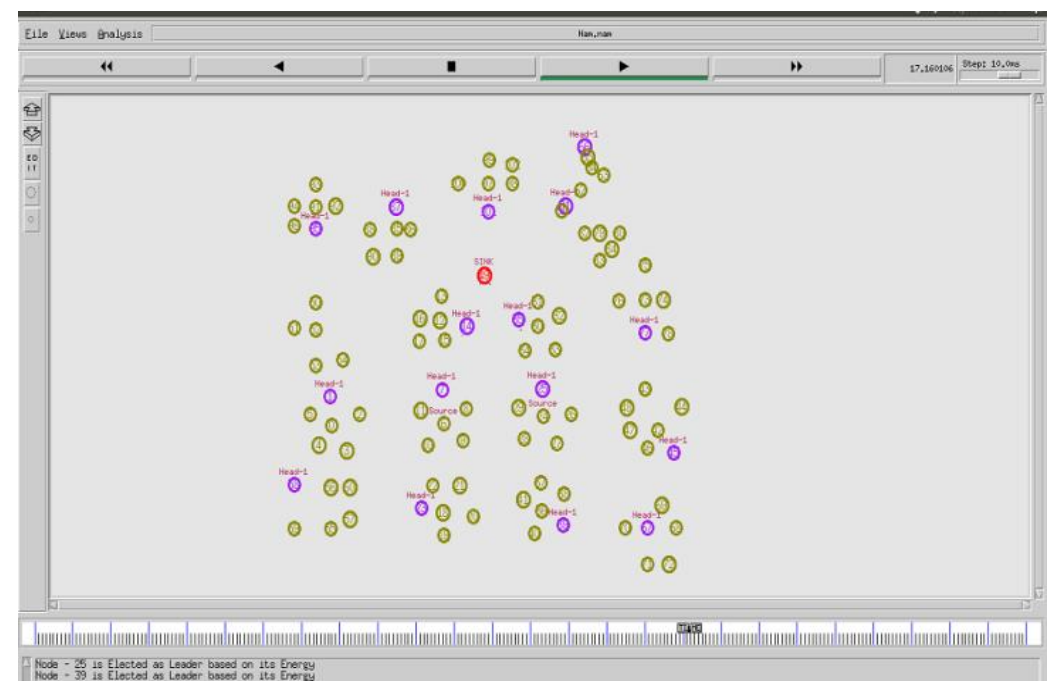

Figure 5: Formation of Cluster and $\mathrm{CH}$ selection

\subsubsection{Energy Calculation}

As shown in Figure 6, all the nodes are grouped into clusters. Energy calculation is done for all the nodes as explained in the above algorithm section. The initial energy is considered between 0.5-1.5 J. Source and destination nodes are fixed by considering the Sink as the destination node.

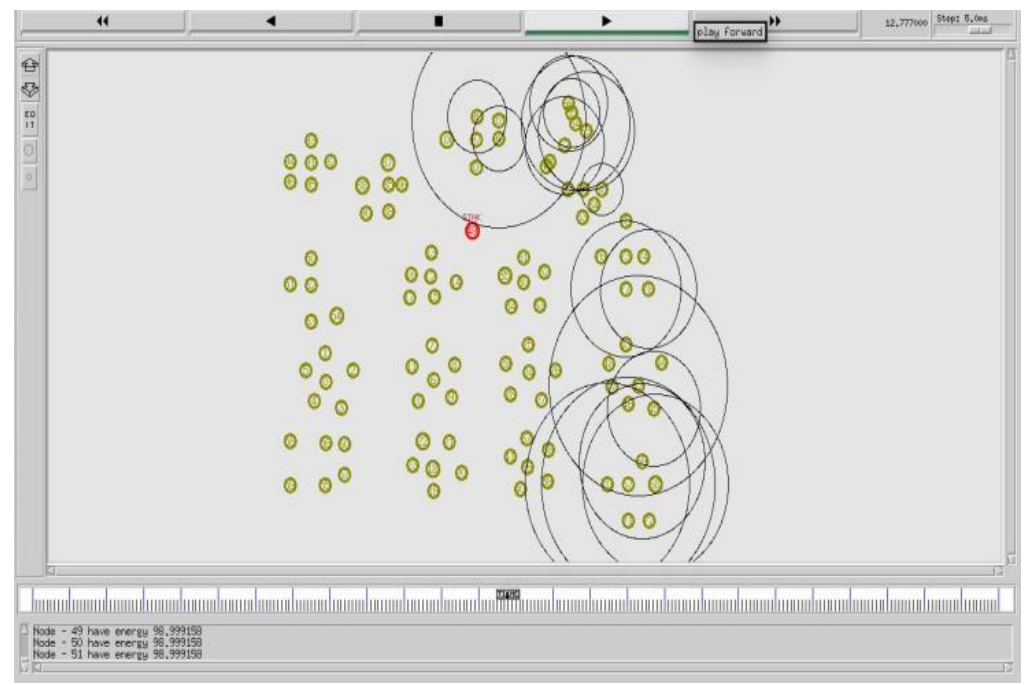

Figure 6: Energy calculation of nodes

\section{Performance Analysis}

The proposed system is implemented using NS-2.35 and compared with HEED and REECHD to compare the network performance in terms of parameters such as energy efficiency, throughput, delay, and PDR. HWSN is considered by deploying 100 nodes on a grid size considered. Following are the parameters considered in the proposed system with graphs and values. 


\subsection{Energy Graph}

As shown in the below Figure. 7, the proposed system uses a clustering algorithm that calculates the $\mathrm{CH}$ based on the high energy. $\mathrm{CHs}$ of all clusters are calculated subsequently by neglecting the cluster whose $\mathrm{CH}$ is already found. $\mathrm{CHs}$ of all clusters in the network are identified.

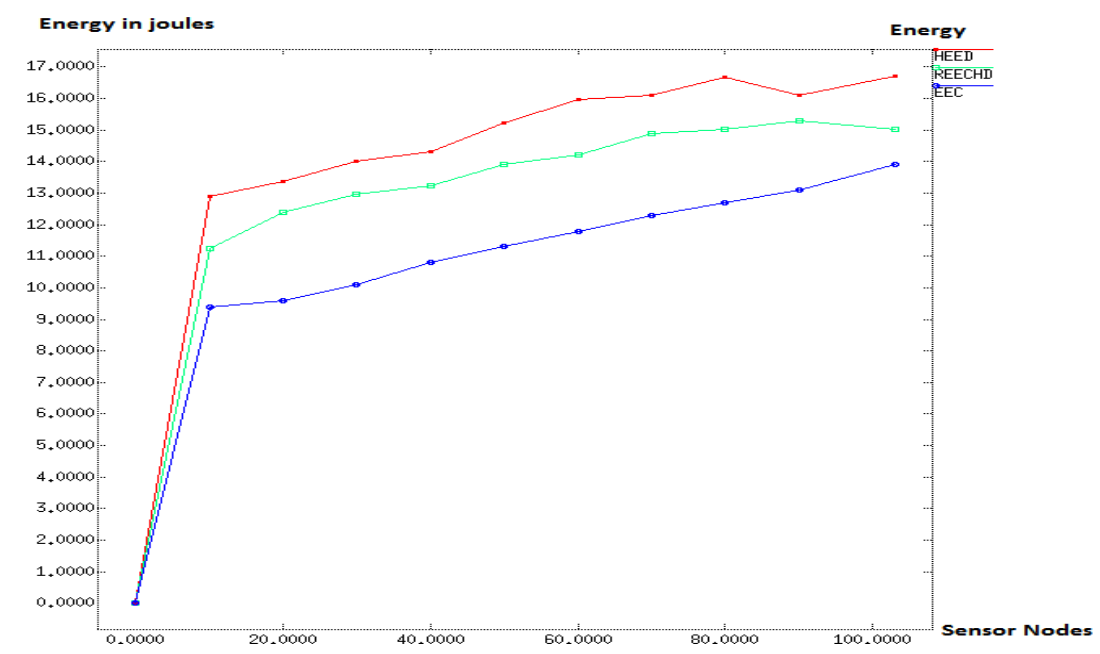

Figure. 7: Energy Graph

During transmission, the particular cluster is considered; $\mathrm{CH}$ is transmitting the data packets to the BS. Whereas other $\mathrm{CHs}$ remain static as the transmission is not taking place within those clusters. This saves the energy for all other clusters as they are not part of a transmission. $\mathrm{CH}$ rotation takes place when the energy of $\mathrm{CH}$ which is transmitting gets depleted. Consumption of energy reduces during $\mathrm{CH}$ selection and transmission. Table 2 shows the values of the proposed system of energy consumption during transmission and compared with the existing system with HEED and REECHD. Energy consumption values are taken by considering the number of sensor nodes on the $\mathrm{X}$-axis and Energy in the joule along the $\mathrm{Y}$-axis. Consumption of energy in the EEC system is 13.9 joules whereas the energy consumed is more in the existing systems.

Table 2: Energy Values of Proposed and Existing Systems

\begin{tabular}{|l|l|l|l|}
\hline $\begin{array}{l}\text { Sensor } \\
\text { Nodes }\end{array}$ & \multicolumn{3}{|c|}{$\begin{array}{c}\text { Energy Consumption } \\
\text { (in joule) }\end{array}$} \\
\hline & HEED[29] & REECHD[30] & EEC(Proposed) \\
\hline 0 & 0 & 0 & 0 \\
\hline 10 & 12.90 & 11.25 & 9.4 \\
\hline 20 & 13.38 & 12.38 & 9.6 \\
\hline 30 & 14.01 & 12.95 & 10.1 \\
\hline 40 & 14.32 & 13.25 & 10.8 \\
\hline 50 & 15.24 & 13.90 & 11.3 \\
\hline 60 & 15.98 & 14.20 & 11.8 \\
\hline 70 & 16.12 & 14.90 & 12.3 \\
\hline 80 & 16.67 & 15.01 & 12.7 \\
\hline 90 & 16.12 & 15.29 & 13.1 \\
\hline 100 & 16.72 & 15.02 & 13.9 \\
\hline
\end{tabular}




\subsection{Packet Delivery Ratio}

Figure 8 shows the PDR of both existing and proposed systems. It is the ratio of no. of packets obtained at the receiver to the total no. of packets including the packet drop. In the proposed system, the PDR is achieved with better value as the MAC layer is tuned up with parameters such as CSMA for detecting the collision and the contention window is maximized for faster transmission.

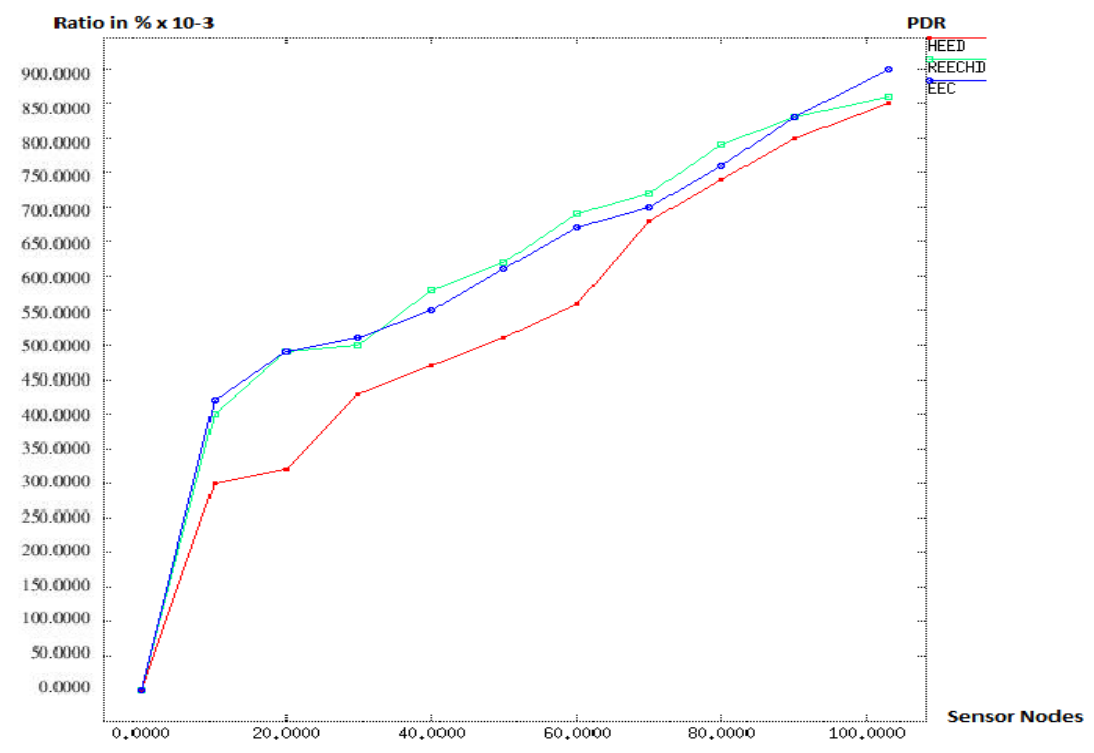

Figure. 8: Packet Delivery Ratio

The physical transmission range is also extended for making the transmission faster. The packet drop is minimized in the proposed system compared to the existing one. Below Table 3 shows the values of the proposed and existing systems of PDR. The proposed system achieves a better packet delivery ratio of about $90 \%$, whereas the existing system has $85 \%$ and $86 \%$. The graph is plotted taking sensor nodes and PDR on X-axis and Y-axis.

Table 3. PDR Values of Proposed and Existing Systems

\begin{tabular}{|l|l|l|l|}
\hline $\begin{array}{l}\text { Sensor } \\
\text { Nodes }\end{array}$ & \multicolumn{3}{|c|}{$\begin{array}{c}\text { PDR } \\
\text { (Ratio in \% x10 }\end{array}$} \\
\hline & HEED[29] & REECHD[30] & EEC(Proposed) \\
\hline 0 & 0 & 0 & 0 \\
\hline 10 & 0.30 & 0.40 & 0.42 \\
\hline 20 & 0.32 & 0.49 & 0.49 \\
\hline 30 & 0.43 & 0.50 & 0.51 \\
\hline 40 & 0.47 & 0.58 & 0.55 \\
\hline 50 & 0.51 & 0.62 & 0.61 \\
\hline 60 & 0.56 & 0.69 & 0.67 \\
\hline 70 & 0.68 & 0.72 & 0.70 \\
\hline 80 & 0.74 & 0.79 & 0.76 \\
\hline 90 & 0.80 & 0.83 & 0.83 \\
\hline 100 & 0.85 & 0.86 & 0.90 \\
\hline & & & \\
\hline
\end{tabular}




\subsection{End to End Delay}

This is the parameter that gives the overall delay in time that the data packets suffer during transmission from source to destination across the network. The overall delay includes queuing delay, propagation delay, and end system processing delays. Packets are lost when it gets delayed longer than the threshold value. Figure. 9 shows End to end delay of both proposed and existing systems. The values of End to End Delay for the proposed and existing system are given in Table 4.

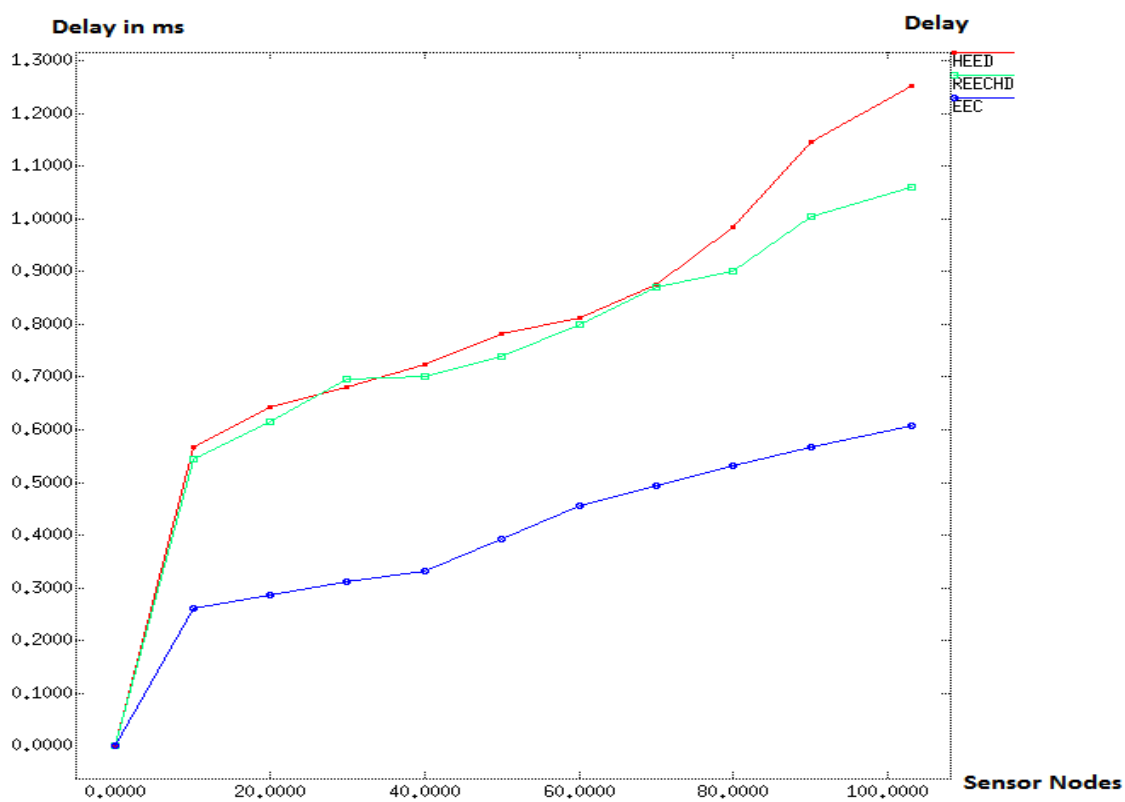

Figure. 9: End to End Delay

Delay in the proposed is minimum compared to the existing system due to the clustering protocol as it selects the $\mathrm{CH}$ at once and using a congestion window based algorithm enhanced at the MAC layer. Delay is reduced by 50\% in the EEC system compared to REECHD which is high that is 1.060 microseconds.

Table 4. Delay Values of Proposed and Existing Systems

\begin{tabular}{|c|c|c|c|}
\hline $\begin{array}{l}\text { Sensor } \\
\text { Nodes }\end{array}$ & \multicolumn{3}{|c|}{$\begin{array}{c}\text { End to End Delay } \\
\text { (In Micro seconds) }\end{array}$} \\
\hline & HEED[29] & REECHD[30] & EEC(Proposed) \\
\hline 0 & 0 & 0 & 0 \\
\hline 10 & 0.567 & 0.545 & 0.260 \\
\hline 20 & 0.642 & 0.615 & 0.287 \\
\hline 30 & 0.681 & 0.695 & 0.312 \\
\hline 40 & 0.723 & 0.700 & 0.332 \\
\hline 50 & 0.782 & 0.740 & 0.392 \\
\hline 60 & 0.812 & 0.800 & 0.456 \\
\hline 70 & 0.876 & 0.870 & 0.493 \\
\hline 80 & 0.985 & 0.900 & 0.532 \\
\hline 90 & 1.145 & 1.005 & 0.567 \\
\hline 100 & 1.253 & 1.060 & 0.608 \\
\hline
\end{tabular}




\subsection{Throughput}

The no. of data packets delivered from source to destination in a specific period time is the Throughput. It is affected by many factors such as routers and cables, congestion, and packet loss. In the proposed system, the number of packets sent is 590. The MAC layer is enhanced with contention window-based algorithm which tunes some of the parameters like the contention window size. Figure 10 shows the throughput graph of both proposed and existing systems by plotting sensor nodes and throughput in $\mathrm{kb} / \mathrm{s}$.

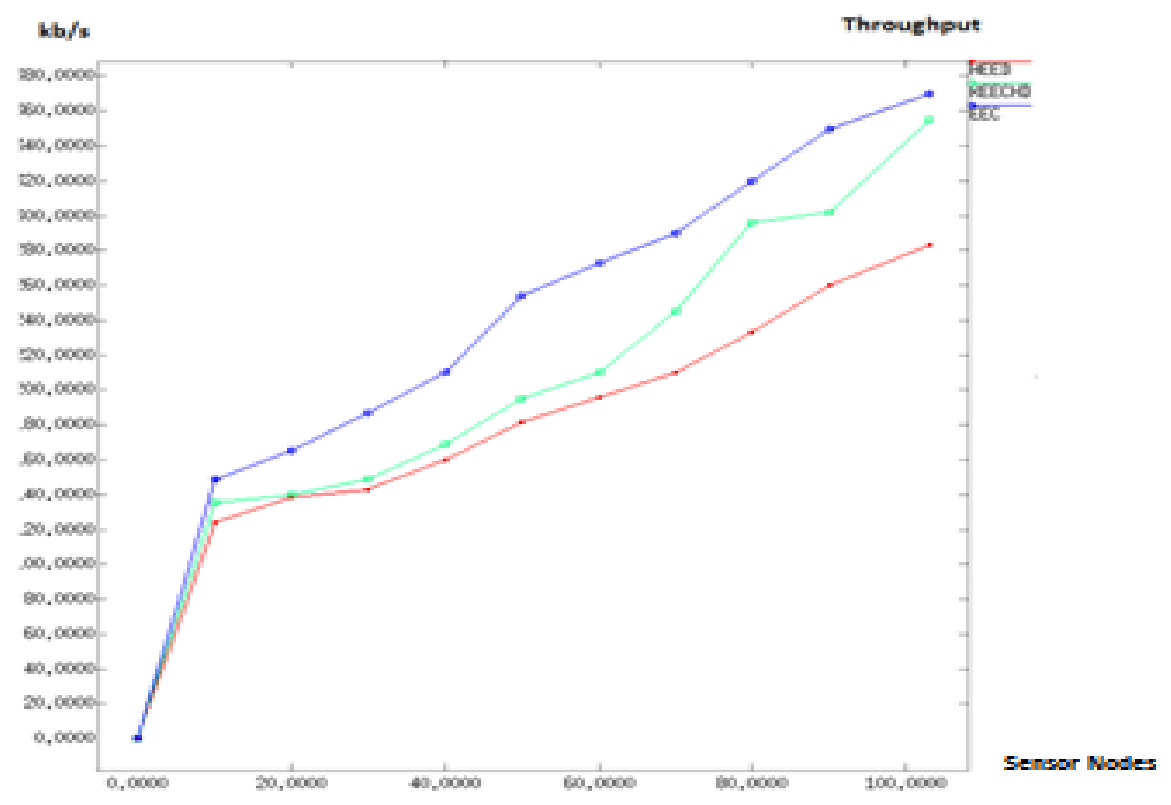

Figure. 10: Throughput

Below Table 5 have throughput values with a comparison with HEED and REECHD. In the EEC system, the decongestion method is incorporated in the MAC layer. Better throughput is achieved in EEC as the system is initiated with a data rate of $11 \mathrm{mbps}$ and frequency of $2.4 \mathrm{GHz}$. EEC system achieves by sending 370 data packets to the sink node.

Table 5. Throughput Values of Proposed and Existing Systems

\begin{tabular}{|c|c|c|c|}
\hline $\begin{array}{c}\text { Sensor } \\
\text { Nodes }\end{array}$ & \multicolumn{3}{|c|}{$\begin{array}{c}\text { Throughput } \\
\text { (kbps) }\end{array}$} \\
\hline & HEED[29] & REECHD[30] & EEC(Proposed) \\
\hline 0 & 0 & 0 & 0 \\
\hline 10 & 124 & 135 & 149 \\
\hline 20 & 138 & 140 & 165 \\
\hline 30 & 143 & 149 & 187 \\
\hline 40 & 160 & 169 & 210 \\
\hline 50 & 182 & 195 & 254 \\
\hline 60 & 196 & 210 & 273 \\
\hline 70 & 210 & 245 & 290 \\
\hline 80 & 233 & 296 & 320 \\
\hline 90 & 260 & 302 & 350 \\
\hline 100 & 283 & 355 & 370 \\
\hline
\end{tabular}


International Journal of Computer Networks \& Communications (IJCNC) Vol.13, No.1, January 2021

\section{Conclusions}

An important research area is saving energy in communication media. Many existing systems having energy-efficient mechanims have been surveyed. It is very difficult to employ a particular type of mechanisms to save energy due to a wide range of network applications. MAC uses many approaches to improve the performance by saving energy. In the proposed system, Energy Efficient Clustering (EEC) is presented for HWSNs which is a clustering protocol that uses $\mathrm{CH}$ rotation. EEC operates in electing the leader and rotation by combining the node higher energy and node induced work. Intra traffic communication is reduced by this $\mathrm{CH}$ selection strategy thus extending the network lifetime. Contention window-based algorithm is enhanced in the MAC layer for decongestion thereby increasing the throughput and reducing the delay.EEC is more energy efficient compared to HEED and REECHD. In the future, many strategies to experiment with various member selections for the formation of cluster by incorporating unequal-sized clustering can be done.

\section{CONFLICTS OF INTEREST}

The authors declare no conflict of interest.

\section{ACKNOWLEDGMENTS}

The authors would like to thank all the staff of the Department of Telecommunication Engineering, Principal, Dr. Ambedkar Institute of Technology for the support given and also thankful to Technical Education Quality Improvement Programme (TEQIP-III) for providing financial support.

\section{REFERENCES}

[1] Anna Forster, "Introduction to Wireless Sensor Networks", John Wiley and Sons: Technology \& Engineering, pp.1-192, 2016.

[2] Bilal Jan, Haleem Farman, Huma Javed, Bartolomeo Montrucchio, Murad Khan, and Shaukat Ali, "Energy Efficient Hierarchial Clustering Approaches in Wireless Sensor Networks: A Survey", Hindawi: Wireless Communications and Mobile Computing, vol. 17, no. 2, pp. 1-14, 2017.

[3] Karanpreet Kaur, Manpreet Singh, Gurdeep Singh, "A survey on Energy efficient Hierarchial clustering algorithm for wireless sensor networks," International Journal of Engineering and Computer Science, vol.6, no. 6, pp. 21849-21853, 2017.

[4] D. Jia, H. Zhu, S. Zou, and P. Hu, "Dynamic cluster head selection method for wireless sensor network," IEEE Sensors Journal, vol. 16, no. 8, pp. 2746-2754, 2016.

[5] H. Farman, H. Javed, J. Ahmad, B. Jan, and M. Zeeshan, "Grid-based hybrid network deployment approach for energy efficient wireless sensor networks," Journal of Sensors, vol.16, no.2, pp. 1-14, 2016.

[6] X. Meng, X. Shi, Z. Wang, S. Wu, and C. Li, "A grid-based reliable routing protocol for wireless sensor networks with randomly distributed clusters," Ad Hoc Networks, vol. 51, no.3, pp. 47-61, 2016.

[7] S. M. Zin, N. B. Anuar, M. L. M. Kiah, and A.-S. K. Pathan, "Routing protocol design for secure WSN: review and open research issues," Journal of Network and Computer Applications, vol.41, no.1, pp. 517-530, 2014.

[8] P. S. Mann and S. Singh, "Energy-efficient hierarchical routing for wireless sensor networks: a swarm intelligence approach," Wireless Personal Communications, vol. 92, no. 2, pp.785-805, 2017.

[9] Đoko Banđur, Branimir Jakšić, Miloš Banđur, Srđan Jović, "An analysis of energy efficiency in Wireless Sensor Networks (WSNs) applied in smart agriculture," Computers and Electronics in Agriculture, vol. 156, no. 1, pp. 500-507, 2018.

[10] W. Heinzelman, J. Kulik, and H. Balakrishnan, "Adaptive protocols for information dissemination in wireless sensor networks," in Proceedings of the 5th ACM/IEEE Mobicom Conference, pp. 174-185, 1999. 
International Journal of Computer Networks \& Communications (IJCNC) Vol.13, No.1, January 2021

[11] Curt Schurgers and Mani B. Srivastava, "Energy efficient routing in wireless sensor networks," MILCOM Proceedings Communications for Network-Centric Operations: Creating the Information Force, vol.1, no. 1, pp. 357-361, 2001.

[12] M. Younis, M. Youssef, and K. Arisha, "Energy-aware routing in cluster-based sensor networks," International Symposium on Modeling, Analysis and Simulation of Computer and Telecommunications Systems, pp. 129-136, 2002.

[13] Jae-Hwan Chang and L. Tassiulas, "Maximum lifetime routing in wireless sensor networks," IEEE/ACM Transactions on Networking, vol. 12, no. 4, pp. 609-619, 2004.

[14] A. Razaque, M. Almiani, M. J. khan, M. N. Kakenuly and Y. Jararweh, "Energy Efficient Medium Access Control Protocol for Wireless Sensor Networks," International Renewable and Sustainable Energy Conference (IRSEC), pp. 1-6, 2018.

[15] R. E. Mohamed, W. R. Ghanem and M. A. Mohamed, "TECEAP: Two-tier era-based clustering energy-efficient adaptive and proactive routing protocol for wireless sensor networks," National Radio Science Conference (NRSC), pp. 187-196, 2018.

[16] V. Accha and S. H. Gupta, "Performance analysis of Wireless Sensor Network MAC protocols using NS-2," International Conference on Computing, Power and Communication Technologies (GUCON), pp. 859-862, 2018.

[17] R. Ngangbam, A. Hossain and A. Shukla, "An improved Clustering Based Hierarchical Protocol for extending Wireless Sensor Network Lifetime - EG LEACH," IEEE International Conference on System, Computation, Automation and Networking (ICSCA), pp. 1-5, 2018.

[18] Liu, Yuxin \& Ota, Kaoru \& Zhang, Kuan \& Ma, Ming \& Xiong, Naixue \& Liu, Anfeng \& Jun Long, "QTSAC: An Energy-Efficient MAC Protocol for Delay Minimization in Wireless Sensor Networks", IEEE Access. pp.8273-8291, 2018.

[19] S. A. Sert, A. Alchihabi and A. Yazici, "A Two-Tier Distributed Fuzzy Logic Based Protocol for Efficient Data Aggregation in Multihop Wireless Sensor Networks," in IEEE Transactions on Fuzzy Systems, vol. 26, no. 6, pp. 3615-3629, 2018.

[20] Alsaafin, Z. A. Aghbari and A. M. Khedr, "Heterogeneous-Aware Distributed Clustering for Wireless Sensor Networks," International Conference on Electro/Information Technology, pp. 0012-0017, 2018.

[21] S. Toor and A. K. Jain, "A new Energy Aware Cluster Based Multi-hop Energy Efficient routing protocol for Wireless Sensor Networks," International Conference on Smart Energy Grid Engineering, pp. 133-137, 2018.

[22] R. Lakshminarayanan, P. Rajendran and M. Thangavel, "Efficient Energy Improvement in Heterogeneous WSNs using Multilevel Network Modeling," International Conference on Emerging Devices and Smart Systems, pp. 60-63, 2018.

[23] U. Kumari, "Investigation: Life-Time and Stability Period in Wireless Sensor Network," International Conference for Convergence in Technology, pp. 1-5, 2018.

[24] S. A. Almogahed and I. A. M. Abdelrahman, "Optimized Distributed Energy Efficient Clustering Scheme for Heterogeneous WSNs," International Conference on Computer, Control, Electrical, and Electronics Engineerin, pp. 1-6, 2018.

[25] El Bhiri, R. Zerououl and A. Jorio, "Spectral classification for a robust clustering in an Heterogenous Wireless Sensor Networks," Renewable Energies, Power Systems \& Green Inclusive Economy, pp. 1$5,2018$.

[26] S. Singh, A. Malik, and R. Kumar, "Energy efficient heterogeneous DEEC protocol for enhancing lifetime in WSNs," International Journal of Engineering Science and Technology, vol.20, no.1, pp.345-353, 2017.

[27] Lakshminarayanan, R, Rajendran, and Thangavel M, "Rotating Energy Efficient Clustering for Heterogeneous Devices (REECHD)," International Conference on Emerging Devices and Smart Systems, pp. 60-63, 2018.

[28] Matteo Micheletti, Leonardo Mostarda, and Andrea Piermarteri, "Rotating Energy Efficient Clustering for Heterogeneous Devices (REECHD)," International Conference on Advanced Information Networking and Applications, pp. 213-220, 2018. 